\title{
Electrochemical Impedance and Complex Capacitance to Interpret Electrochemical Capacitor
}

\author{
Masayuki Itagaki, Satoshi Suzuki, Isao Shitanda, and Kunihiro Watanabe
}

\begin{abstract}
Department of Pure and Applied Chemistry, Faculty of Science and Technology, Tokyo University of Science (Noda, Chiba 278-8510, Japan)
\end{abstract}

Received February 12, 2007 ; Accepted May 14, 2007

\begin{abstract}
The electrochemical impedance $Z$ and complex capacitance $C$ for typical equivalent circuits were summarized systematically in order to support the frequency domain analysis of electric double layer capacitance (EDLC). In the present paper, the impedance and the complex capacitance were calculated in the case that the electrochemical response of porous electrode was presented by a transmission line model (TLM). The loci of $Z$ and $C$ on the complex were divided into the lumped constant and distributed constant ranges in low and high frequency ranges, respectively. The complex capacitance plot was superior to obtain the capacitance of porous electrode than the impedance plot.
\end{abstract}

Key Words : Electrochemical Impedance Spectroscopy, Complex Capacitance, Transmission Line Model, Electrochemical Capacitor.

\section{Introduction}

Electrochemical impedance spectroscopy (EIS) is an analytical tool for heterogeneous interface in the frequency domain by the comparison of sinusoidal input potential (current) signal with output current (potential) signal. The principle of EIS has been developed with the measurements of dielectric constant and resistivity of solutions at the middle of $20^{\text {th }}$ century. For example, Cole and co-worker ${ }^{1,2)}$ determined the dielectric constants of the solutions by complex plane plot and derived various equations to present the dielectric relaxation.

In recent years, the measurement of electrochemical impedance has become easily by the development of frequency response analyzer and the software. Therefore, the EIS has been applied to investigations of energy conversion devices like fuel cell, lithium ion battery, electrochemical double layer capacitor (EDLC). ${ }^{3-14}$ Regarding EIS analysis on EDLC, Taberna et al. ${ }^{5,6)}$ investigated the influences of electrolyte and electrode material on the performance of EDLC by the measurement of electrochemical impedance. Eikerling et al. ${ }^{7)}$ proposed the electrode model of EDLC whose electrode was active carbon with nano-micro pores, and carried out the simulation for impedance spectra. In addition, some researchers ${ }^{5,6,12-14}$ analyzed the performance of energy conversion devices by the complex capacitance plots. Jang et al. ${ }^{14)}$ analyzed the leakage current of EDLC by complex capacitance plots. However, there is no paper to summarize the relations between the complex capacitance spectra and the various equivalent circuits. In the present paper, electrochemical impedance and complex capacitance corresponding to typical equivalent circuits involving a transmission line model (TLM) are calculated and the important points to interpret the spectra are discussed.

\section{Theory}

\section{1 Electrochemical impedance}

EIS is non-steady state method to calculate transfer function (impedance or admittance) of electrode reaction by the comparison of output with input signals in the frequency domain. The transfer function is decided by various factors such as charge transfer, diffusion, reaction mechanism, electrode structure, thus it can be used to analyze reaction and condition of electrode. In general, impedance $Z$ is expressed as a function of alternate current $I$ and potential $E$ as follows,

$$
Z=\frac{E}{I}
$$

where,

$$
\begin{gathered}
E=E_{m} \exp (j \omega t) \\
I=I_{m} \exp \{j(\omega t+\varphi)\}
\end{gathered}
$$

The impedance $Z$ is expressed as follows,

$$
Z=|Z| \exp (j \theta),|Z|=\frac{E_{m}}{I_{m}}, \theta=-\varphi
$$

where $E_{m}, I_{m}$ and $f$ are voltage amplitude, current amplitude and phase difference, respectively. Impedance $Z$ is expressed by the absolute value $|Z|$ and phase shift $\theta$ from Eq. (4). In addition, the impedance is often represented by the real part $Z^{\prime}$ and imaginary part $Z^{\prime \prime}$ as a complex number.

$$
Z=Z^{\prime}+j Z^{\prime \prime}
$$

\section{2 Complex capacitance}

Total current $I$ flowing in dielectric material is com- 
posed of the current $I_{G}$ to charge the polarity and the leak current $I_{Q}$,

$$
I=I_{G}+I_{Q}=G_{s s} E+\frac{d\left(C_{s s} E\right)}{d t}
$$

where $G_{s s}$ and $C_{s s}$ are conductance and capacitance at steady-state, respectively. Eq. (6) is transformed by using Eq. (2) as follows,

$$
\begin{gathered}
I=Y E \\
Y=Y^{\prime}+j Y^{\prime \prime}, Y^{\prime}=G_{s s}, Y^{\prime \prime}=\omega C_{s s}
\end{gathered}
$$

where $Y$ is defined as complex admittance. The current has a following relation with the charge $Q$ since the $Q$ can be expressed by sinusoidal equation.

$$
I=\frac{d Q}{d t}=j \omega Q
$$

From Eqs. (7) and (9),

$$
\frac{Q}{E}=\frac{Y}{j \omega}=C_{s s}-j \frac{G_{s s}}{\omega}
$$

Since $Q=C E$,

$$
C=C^{\prime}+j C^{\prime \prime}, C^{\prime}=C_{s s}, C^{\prime \prime}=-G_{s s} / \omega
$$

$C^{\prime \prime}$ is related to the dielectric loss. The impedance is inversely proportionate to the admittance.

$$
Z=\frac{1}{Y}
$$

From Eq. (12), the complex capacitance is assessed by following equation.

$$
\begin{gathered}
C=\frac{1}{j \omega Z}=C^{\prime}+j C^{\prime \prime}, C^{\prime}=-\frac{Z^{\prime \prime}}{\omega\left(Z^{\prime 2}+Z^{\prime \prime 2}\right)^{\prime}}, \\
C^{\prime \prime}=-\frac{Z^{\prime}}{\omega\left(Z^{\prime 2}+Z^{\prime \prime 2}\right)}
\end{gathered}
$$

\section{1 Equivalent circuit}

\section{Results and Discussion}

An electrode/electrolyte interface can be presented by equivalent circuit involving some electronic elements when it is analyzed by EIS. Typical equivalent circuits in electrochemical measurement are shown in Fig. 1. The simplest equivalent circuit for the ideal polarization electrode without Faradaic process is series of the electric double layer capacitance $C_{d l}$ and the solution resistance $R_{\text {sol }}$ (Fig. 1 (a)). When the charge transfer resistance $R_{c t}$ is assumed for the electrode with Faradaic process, the equivalent circuit involves the parallel of $R_{c t}$ and $C_{d l}$ (Fig. 1 (b)). In data analysis of electrochemical impedance, every elements are calculated by curve-fitting of experimental results on the basis of the equivalent circuit. Therefore, it is necessary to know the relation between the equivalent circuit and the impedance locus in detail. (a)

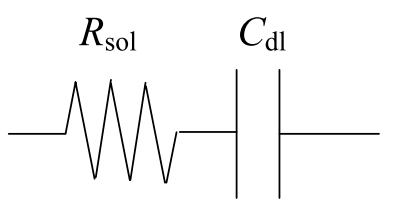

(b)

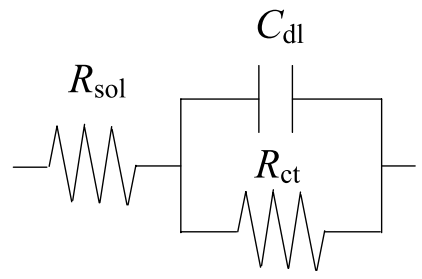

Fig. 1 Typical equivalent circuits of electrode/electrolyte interfaces (a) without Faradaic process and (b) with Faradaic process.

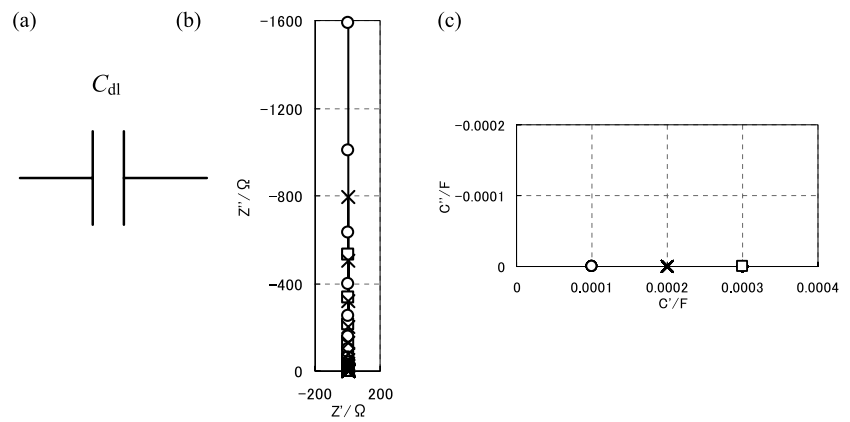

Fig. 2 (a) Equivalent circuit consisting of $C_{d l}$. (b) The calculated results of electrochemical impedance. (c) The calculated results of complex capacitance. The values of parameters are as follows: $\bigcirc: C_{d l}=1.0 \times 10^{-4} \mathrm{~F}, \times: C_{d l}=$ $2.0 \times 10^{-4} \mathrm{~F}, \square: C_{d l}=3.0 \times 10^{-4} \mathrm{~F}$.

\section{2 Impedance and complex capacitance of various} equivalent circuits

3.2. $1 C_{d l}$

3. 2. 1. 1 Impedance The equivalent circuit is shown in Fig. 2 (a), when the impedance of electrode/ electrolyte interface is represented by only $C_{d l}$. In this case, the real and imaginary parts of impedance are as follows.

$$
Z^{\prime}=0 \quad Z^{\prime \prime}=-\frac{1}{j \omega C_{d l}}
$$

The calculated results of electrochemical impedance with variations of $C_{d l}$ are shown in Fig. 2 (b). The locus of impedance is described on minus part of the imaginary axis on complex plane and converges at origin in high frequency range. The absolute value of impedance decreases with the increase of $C_{d l}$.

3. 2. 1. 2 Complex capacitance The complex capacitance was calculated by Eq (13) from $Z$.

$$
C^{\prime}=C_{d l} \quad C^{\prime \prime}=0
$$


(a)

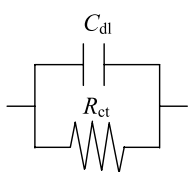

(b)

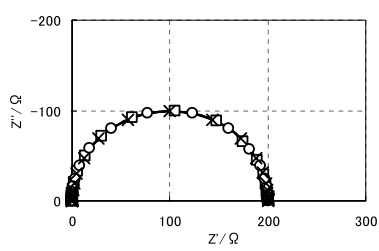

(c)

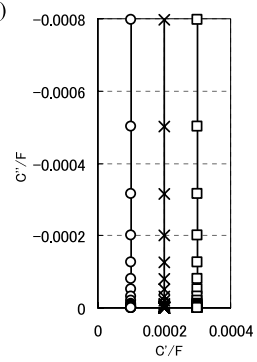

Fig. 3 (a) Parallel circuit consisting of $C_{d l}$ and $R_{c t}$. (b) The calculated results of electrochemical impedance. (c) The calculate results of complex capacitance. The values of parameters are as follows: $R_{c t}=200 \Omega, \bigcirc: C_{d l}=1.0 \times 10^{-4} \mathrm{~F}$, $\times: C_{d l}=2.0 \times 10^{-4} \mathrm{~F}, \square: C_{d l}=3.0 \times 10^{-4} \mathrm{~F}$. The frequency range of (b) is $10 \mathrm{mHz}-10 \mathrm{kHz}$, and that of (c) is $10 \mathrm{mHz}-1$ $\mathrm{Hz}$.

Calculated results of complex capacitance are shown in Fig. 2 (c). All plots of $C$ gather the value of $C_{d l}$ on real axis.

\section{2. 2 Parallel circuit of $\boldsymbol{R}_{c t}$ and $C_{d l}$}

3. 2. 2. 1 Impedance The equivalent circuit is shown in Fig. 3 (a), and the real and imaginary parts of $Z$ are as follows.

$$
Z^{\prime}=\frac{R_{c t}}{1+\omega^{2} R_{c t}^{2} C_{d l}^{2}} \quad Z^{\prime \prime}=-\frac{\omega R_{c t}^{2} C_{d l}}{1+\omega^{2} R_{c t}^{2} C_{d l}^{2}}
$$

Furthermore, the low frequency limiting value of impedance at $\omega \rightarrow 0$ are as follows.

$$
Z_{\omega \rightarrow 0}^{\prime}=R_{c t} \quad Z_{\omega \rightarrow 0}^{\prime \prime}=0
$$

The calculated results of electrochemical impedance with variation of $C_{d l}$ are shown in Fig. 3 (b). The impedance describes the locus of semi-circle on complex plane. In Fig. 3 (b), the impedance converges at origin and $R_{c t}$ in high and low frequency ranges, respectively. Though the value of $C_{d l}$ is charged, the shape of locus is identical. Since $R_{c t} C_{d l}$ can be obtained from the frequency $f$ at maximum of imaginary part $\left(R_{c t} C_{d l}=1 / 2 \pi f\right)$, the $C_{d l}$ can be calculated from the values of $R_{c t} C_{d l}$ and $R_{c t}$. In Fig. 3 (c), the shape of impedance spectrum is not changed. And the time constant of dielectric relaxation $\left(1 / \omega=t_{1}=\right.$ $R_{c t} C_{d l}$ ) could be obtained from peak of semi-circle. The peak frequency decreases and time constant of dielectric relaxation increases with the increase of $C_{d l}$.

\section{2. 2. 2 Complex capacitance The complex} capacitance in the case of parallel circuit $R_{c t}$ and $C_{d l}$ are as follows.

$$
C^{\prime}=C_{d l} \quad C^{\prime \prime}=-\frac{1}{\omega R_{c t}}
$$

The calculated results of complex capacitance with variation of $R_{c t}, C_{d l}$ are shown in Fig. 3 (c). Complex capacitance describes the locus in parallel with the imaginary axis on complex plane and converged at $C_{d l}$ on real axis in high frequency range. Contrary to $Z$ in Fig. 3 (b),

(a)

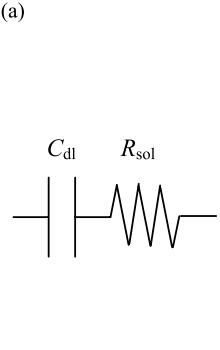

(b)

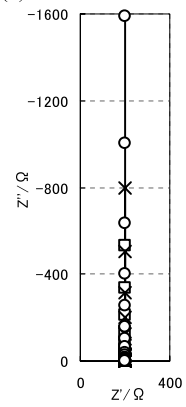

(c)

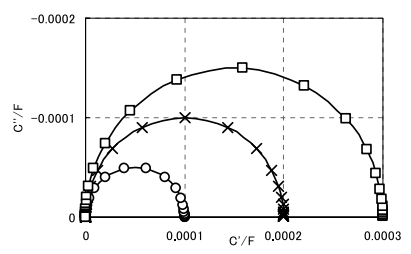

Fig. 4 (a) Series circuit consisting of $C_{d l}$ and $R_{\text {sol. }}$ (b) The calculated results of electrochemical impedance. (c) The calculated results of complex capacitance. The values of parameters are as follows: $R_{\text {sol }}=200 \Omega, \bigcirc: C_{d l}=1.0 \times 10^{-4} \mathrm{~F}$, $\times: C_{d l}=2.0 \times 10^{-4} \mathrm{~F}, \square: C_{d l}=3.0 \times 10^{-4} \mathrm{~F}$. The frequency range of (b) is $10 \mathrm{mHz}-1 \mathrm{~Hz}$, and that of (c) is $10 \mathrm{mHz}-10$ $\mathrm{kHz}$.

the value of $C_{d l}$ can be determined directry in Fig. 3 (c).

3. 2. 3 Series circuit of $\boldsymbol{R}_{s o l}$ and $C_{d l}$

3. 2. 3. 1 Impedance The impedance of equivalent circuit shown in Fig. 4 (a) is as follows.

$$
Z^{\prime}=R_{\text {sol }} \quad Z^{\prime \prime}=-\frac{1}{j \omega C_{d l}}
$$

The calculated results of impedance with variation of $C_{d l}$ are shown in Fig. 4 (b). In Fig. 4 (b), impedance shows vertical straight line and converges at value of $R_{\text {sol }}$ on real axis in high frequency range.

3. 2. 3. 2 Complex capacitance The complex capacitance was calculated by Eqs. (13) and (19).

$$
C^{\prime}=\frac{C_{d l}}{1+\omega^{2} C_{d l}^{2} R_{\text {sol }}} \quad C^{\prime \prime}=-\frac{\omega C_{d l}^{2} R_{\text {sol }}}{1+\omega^{2} C_{d l}^{2} R_{\text {sol }}}
$$

Furthermore, the low frequency limiting value of capacitance are as follows.

$$
C_{\omega \rightarrow 0}^{\prime}=C_{d l} \quad C_{\omega \rightarrow 0}^{\prime \prime}=0
$$

Calculated results of complex capacitance with variation of $C_{d l}$ are shown in Fig. 4 (c). Complex capacitance describes locus of semi-circle on complex plane. The locus converges at origin and $C_{d l}$ in high and low frequency ranges, respectively. The time constant of the dielectric relaxation $R_{s o l} C_{d l}$ can be obtained from the frequency at maximum point imaginary part $\left(R_{s o l} C_{d l}=\right.$ $1 / 2 \pi f)$.

3. 2. $4 R_{\text {sol }}$ with parallel circuit $\boldsymbol{R}_{c t}$ and $C_{d l}$

3. 2. 4. 1 Impedance The impedance of equivalent circuit in Fig. 5 (a) is as follows.

$$
Z^{\prime}=R_{s o l}+\frac{R_{c t}}{1+\omega^{2} R_{c t}^{2} C_{d l}^{2}} \quad Z^{\prime \prime}=-\frac{\omega R_{c t}^{2} C_{d l}}{1+\omega^{2} R_{c t}^{2} C_{d l}^{2}}
$$

The calculated results of electrochemical impedance with variation of $C_{d l}$ are shown in Fig. 5 (b). The locus of 

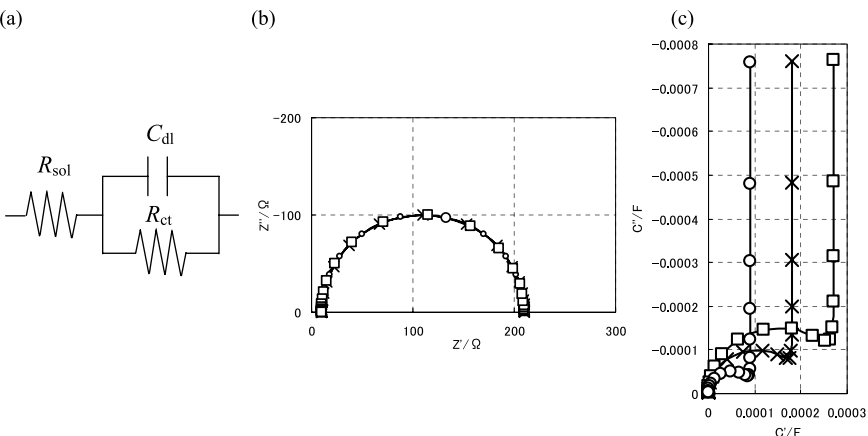

Fig. 5 (a) Equivalent circuit consisting of $R_{\text {sol }}$ and parallel of $C_{d l}$ and $R_{c t}$. (b) The calculated results of electrochemical impedance. (c) The calculated results of complex capacitance. The values of parameters are as follows: $R_{\text {sol }}=$ $10 \mathrm{O}, R_{c t}=200 \Omega, \bigcirc: C_{d l}=1.0 \times 10^{-4} \mathrm{~F}, \times: C_{d l}=2.0 \times 10^{-4} \mathrm{~F}$, $\square: C_{d l}=3.0 \times 10^{-4} \mathrm{~F}$. The frequency range of (b) is $10 \mathrm{mHz}-$ $10 \mathrm{kHz}$, and that of (c) is $10 \mathrm{mHz}-1 \mathrm{~Hz}$.

impedance is semi-circle on complex plane, and converges at $R_{\text {sol }}$ and $R_{\text {sol }}+R_{c t}$ in high and low frequency ranges, respectively. In Fig. 5 (b), the shape of semi-circle does not depend on the value of $C_{d l}$.

3. 2. 4. 2 Complex capacitance

The complex capacitance was calculated by Eqs. (13) and (22).

$$
\begin{gathered}
C^{\prime}=\frac{R_{c t}^{2} C_{d l}}{\left(R_{c t}+R_{s o l}\right)^{2}+\omega^{2} C_{d l}^{2} R_{c t}^{2} R_{s o l}^{2}} \\
C^{\prime \prime}=-\frac{R_{c t} R_{s o l}+\omega^{2} C_{d l}^{2} R_{c t}^{2} R_{\text {sol }}}{\omega\left(R_{c t}+R_{s o l}\right)^{2}+\omega^{3} C_{d l}^{2} R_{c t}^{2} R_{s o l}^{2}}
\end{gathered}
$$

Furthermore, the low frequency limiting value of capacitance are as follows.

$$
C_{\omega \rightarrow 0}^{\prime}=-\frac{C_{d l}}{\left(R_{c t}+R_{s o l}\right)^{2} / R_{c t}^{2}} \quad C_{\omega \rightarrow 0}^{\prime \prime}=\infty
$$

The calculated results of complex capacitance with variation of $C_{d l}$ are shown in Fig. 5 (c). Complex capacitance describes locus of semi-circle in high frequency range and locus at straight line in parallel with the imaginary axis in low frequency range. In Fig. 5 (c), the diameter of semi-circle increases with the increase of $C_{d l}$, and the value extrapolated from the semi-circle to the real axis in the direction toward low frequency is matched with the value of $C_{d l}$. On the other hand, the value extrapolated from the straight line to real axis is $C_{d l} R_{c t}^{2} /\left(R_{c t}+R_{s o l}\right)^{2}$. Therefore, $C_{d l}$ can be determined from the complex capacitance plot when the $R_{\text {sol }}$ and $R_{c t}$ are known.

\section{3 The impedance of cylindrical electrode by TLM}

When the electrochemical surface is not flat and the shape is presented by three-dimensional electrode, the solution resistance is not uniform and the current distributes on the electrode. The electrochemical property of this kind of electrode could be expressed by TLM. Since the activated carbon used for EDLC has porous structure, TLM can be applied the analysis of electrode structure and performance of EDLC. We presented the elec- (a)

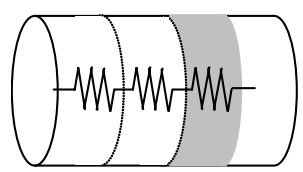

(b)

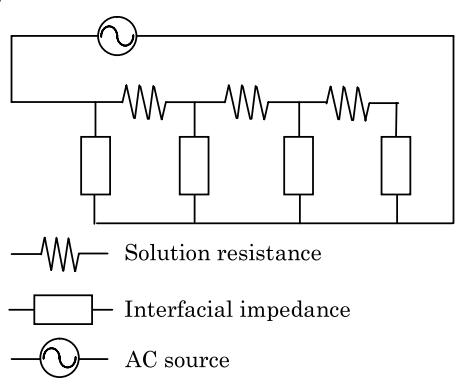

Fig. 6 (a) Scheme of cylindrical electrode. (b) Distributed constant equivalent circuit of cylindrical electrode.

trode surface as inner wall of cylindrical electrode in Fig. 6 (a) in order to simplify the porous electrode. In this case, the equivalent circuit is presented by distributedconstant typed depicted in Fig. 6 (b).

Levie $^{15)}$ solved theoretical formula of impedance presented by TLM as follows.

$$
\begin{gathered}
Z=\sqrt{R_{\text {sol }}^{*} Z^{*}} \operatorname{coth} \sqrt{\frac{R_{\text {sol }}^{*}}{Z^{*}}} X \\
Z^{*}=\frac{Z^{* *}}{L} \\
R_{\text {sol }}^{*}=\frac{\rho}{A}
\end{gathered}
$$

In these equations, $X$ is depth of a pore, $Z^{* *}$ is impedance per unit area, $Z^{*}$ is impedance per unit length, $L$ is circumference of a pore, $R_{\text {sol }}^{*}$ is solution resistance per unit length, $\rho$ is specific resistance, and $A$ is cross-section area of a pore. And $Z^{* *}$ without Faradaic process (blocking electrode) is represented by Eq. (28).

$$
Z^{* *}=\frac{1}{j \omega C_{d l}^{* *}}
$$

$Z^{* *}$ including Faraday process such as redox capacitor is represented by formula (29),

$$
Z^{* *}=\frac{R_{c t}^{* *}}{1+j \omega R_{c t}^{* *} C_{d l}^{* *}}
$$

$R_{c t}^{* *}$ is charge transfer resistance per unit area, $C_{d l}^{* *}$ is capacitance per unit area. Maclaurin expansion of $\operatorname{coth} x$ is

$$
\operatorname{coth} x=\frac{1}{x}+\frac{x}{3}-\frac{x^{3}}{45}+\frac{2 x^{5}}{945}+\cdots, 0<|x|<\pi
$$

In the case that $x \ll 1$, Eq. (30) can be simplified as follows.

$$
\operatorname{coth} x \approx \frac{1}{x}+\frac{x}{3}
$$

Assumption that $x \ll 1$ corresponds to $\omega \rightarrow 0$ in impedance spectrum. Therefore, Levie's formula in low fre- 
quency range can be written as follows.

$$
Z \approx \frac{Z^{*}}{X}+\frac{R_{s o l}^{*} X}{3}
$$

\section{4. 1 TLM without Faradaic process (blocking electrode)}

3. 4. 1. 1 Impedance The equivalent circuit is shown in Fig. 7 (a). The impedance is presented by Eqs. (25) - (28). The calculated results of electrochemical impedance are shown in Figs. 7 (b) and (c). Impedance describes straight line of 45 degree to real axis in high frequency range and vertical line in low frequency range on complex plane. The locus of impedance in the low frequency range is the feature of blocking electrode without Faradaic process, and its equation is derived from Eq. (32).

$$
Z_{\omega \rightarrow 0}^{\prime}=\frac{R_{s o l}^{*} X}{3} \quad Z_{\omega \rightarrow 0}^{\prime \prime}=-\frac{1}{\omega X L C_{d l}^{* *}}
$$

In the low frequency range, the real part of impedance takes a constant value $\frac{R_{s o l}^{*} X}{3}$, where $R_{s o l}^{*} X$ corresponds the solution resistance from inlet to bottom inside cylindrical pore. Beside, the imaginary part increases with the decrease of $\omega$. In Eq. (33) $X L C_{d l}^{* *}$ is the capacitance of whole surface of pore inner wall because $X L$ means its surface area. The 45 degree straight line in the high frequency range originates from the current distribution inside pore. The value of $Z^{\prime}$ at transition point between these ranges is approximately $\frac{R_{s o l}^{*} X}{3}$, and it increases with the $R_{\text {sol }}^{*}$ in Fig. 7 (b). The value of $Z^{\prime}$ is identical with the variation of $C_{d l}^{* *}$ since the values of $R_{s o l}^{*}$ and $X$ are constants in Fig. 7(c), and the curve-fitting with the software is necessary to determine the $C_{d l}$.

3. 4. 1. 2 Complex capacitance The calculated results of complex capacitance are shown in Figs. 7 (d) and (e). Complex capacitance describes the locus of 45 degree to real axis in high frequency range and locus of semi-circle in low frequency range on complex plane. Capacitance converges at $X L C_{d l}^{* *}$ on real axis in low frequency range. Complex capacitance is derived from Eqs. (13) and (25) as follows.

$$
C=\frac{1}{j \omega \sqrt{R_{s o l}^{*} Z^{*}}} \tanh \sqrt{\frac{R_{s o l}^{*}}{Z^{*}}} X
$$

The limiting value of capacitance at $\omega \rightarrow 0$ is calculated as follows.

$$
C_{\omega \rightarrow 0}^{\prime}=X L C_{d l}^{* *} \quad C_{\omega \rightarrow 0}^{\prime \prime}=0
$$

In Fig. 7 (d), the shape of complex capacitance is identical. The time constant of charge/discharge $\left(R_{s o l} C_{d l}\right)$ can be obtained from the maximum peak of semi-circle. ${ }^{14)}$ The time constant increases with the increase of $R_{s o l}^{*}$. In Fig. 7 (e), the low frequency limiting value $C_{\omega \rightarrow 0}^{\prime}$ increas-
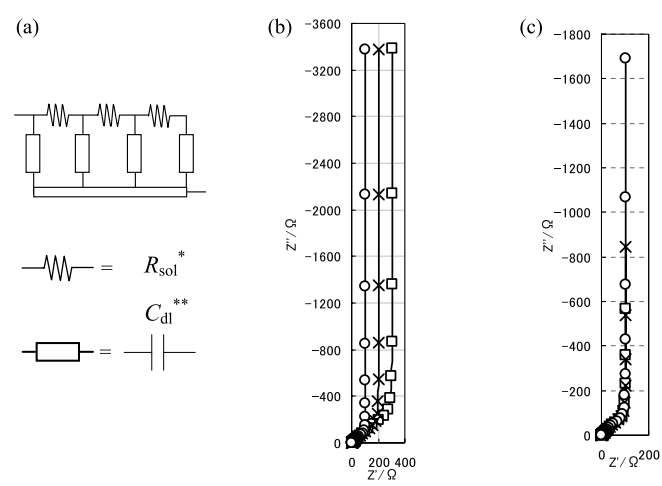

(d)

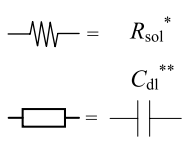

(e)
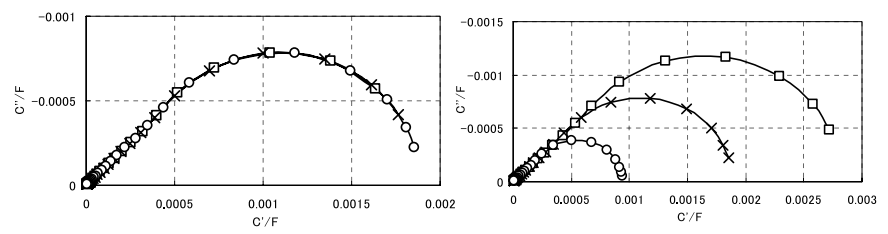

Fig. 7 (a) Equivalent circuit with TLM without Faradaic process. The calculated results of electrochemical impedance with the variations of (b) solution resistance and (c) double layer capacitance. The calculated results of complex capacitance with the variation of (d) solution resistance and (e) double layer capacitance. The values of parameters in (b) and (d) are as follows: $X=1 \mathrm{~cm}, L=\pi \mathrm{cm}$, $C_{d l}^{* *}=5.0 \times 10^{-5} \mathrm{~F} / \mathrm{cm}^{2}, \bigcirc: \rho=80 \mathrm{O} \mathrm{cm}, \times: \rho=160 \Omega \mathrm{cm}, \square$ $: \rho=240 \mathrm{O} \mathrm{cm}$. The values in (c) and (e) are as follows: $X=$ $1 \mathrm{~cm}, L=\pi \mathrm{cm}, \rho=80 \Omega \mathrm{cm}, \bigcirc: C_{d l}^{* *}=1.0 \times 10^{-4} \mathrm{~F} / \mathrm{cm}^{2}, \times$ : $C_{d l}^{* *}=2.0 \times 10^{-4} \mathrm{~F} / \mathrm{cm}^{2}, \square: C_{d l}^{* * *}=3.0 \times 10^{-4} \mathrm{~F} / \mathrm{cm}^{2}$. The frequency range is $100 \mathrm{mHz}-10 \mathrm{kHz}$.

es with the increase of $C_{d l,}^{* *}$ where $C_{\omega \rightarrow 0}^{\prime}=X L C_{d l}^{* *}$ in Eq. (35). The calculated values of $C_{\omega \rightarrow 0}^{\prime}$ were $1.0 \times 10^{-3}, 2.0 \times$ $10^{-3}, 3.0 \times 10^{-3} \mathrm{~F}$, where the values of $C_{d l}^{* *}$ were $1.0 \times 10^{-3}$, $2.0 \times 10^{-3}, 3.0 \times 10^{-3} \mathrm{~F} / \mathrm{cm}^{2}$, respectively, $X$ is $3 \mathrm{~cm}$, and $L$ is $1 \mathrm{~cm}$. These results agree with the loci in Fig. 7 (e). $X L$ and $X L C_{d l}^{* *}$ represent the whole surface area and the whole capacitance, respectively, of inner wall of electrode. From the complex capacitance plot of blocking electrode with TLM, the whole electrode capacitance can be determined directly.

\section{5. 1 TLM including Faradaic process}

3. 5. 1. 1 Impedance The equivalent circuit is shown in Fig. 8 (a). The limiting value of impedance at $\omega$ $\rightarrow 0$ were written from Eqs. (25) and (29).

$$
Z_{\omega \rightarrow 0}^{\prime}=\frac{R_{c t}^{* *}}{X L}+\frac{R_{s o l}^{*} X}{3} \quad Z_{\omega \rightarrow 0}^{\prime \prime}=0
$$

The calculated results of electrochemical impedance with variations of $R_{s o l}^{*}, R_{c t}^{* * *}$ and $C_{d l}^{* *}$ are shown in Figs. 8 (b), (c) and (d), respectively. The impedance describes the locus of 45 degree to real axis in high frequency range and locus of semi-circle in low frequency area. It was reported that impedance with TLM allowed the information regarding the electrode structure and interfacial impedance in high and low frequency ranges, respectively. ${ }^{11)} Z^{\prime}$ at the transition frequency between 
(a)

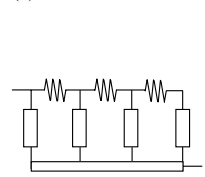

(b)
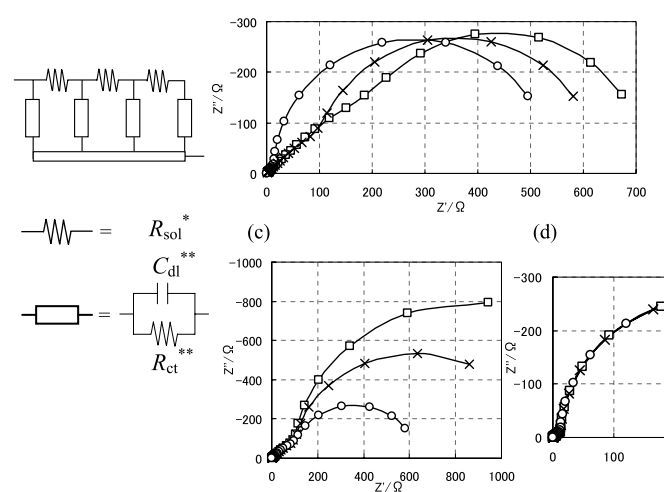

(d)

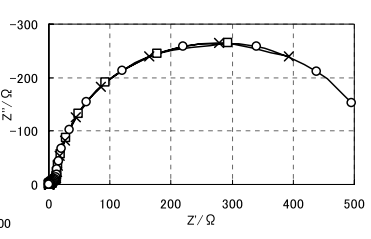

(e)

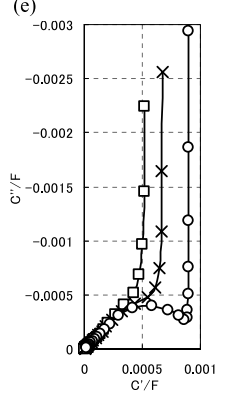

(f)

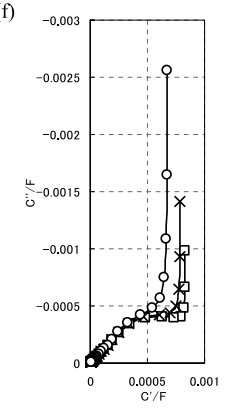

(g)

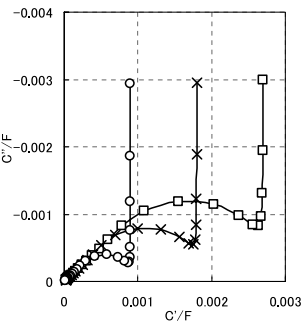

Fig. 8 (a) Equivalent circuit with TLM including Faradaic process. The calculated results of electrochemical impedance with variations of (b) solution resistance, (c) charge transfer resistance and (d) double layer capacitance. The calculated results of complex capacitance with the variations of (e) solution resistance, (f) charge transfer resistance and $(\mathrm{g})$ double layer capacitance. The values of parameters in (b) and (e) are as follows: $X=1 \mathrm{~cm}, L=\pi \mathrm{cm}$, $R_{c t}^{* *}=5000 \mathrm{O} \mathrm{cm}^{2}, C_{d l}^{* *}=1.0 \times 10^{-4} \mathrm{~F} / \mathrm{cm}^{2}, \bigcirc: \rho=10 \mathrm{O} \mathrm{cm}$, $\times: \rho=80 \Omega \mathrm{cm}, \square: \rho=160 \mathrm{O} \mathrm{cm}$. The parameters in (c) and (f) are as follows: $X=1 \mathrm{~cm}, L=\pi \mathrm{cm}, \rho=80 \mathrm{O} \mathrm{cm}$, $C_{d l}^{* *}=1.0 \times 10^{-4} \mathrm{~F} / \mathrm{cm}^{2}, \bigcirc: R_{c t}^{* *}=5000 \mathrm{O} \mathrm{cm}^{2}, \times: R_{c t}^{* *}=10000$ $\mathrm{O} \mathrm{cm}^{2}, \square: R_{c t}^{* *}=15000 \mathrm{O} \mathrm{cm}^{2}$. The parameters in (d) and (g) are as follows: $X=1 \mathrm{~cm}, L=\pi \mathrm{cm}, \rho=10 \mathrm{O} \mathrm{cm}, R_{c t}^{* *}=5000$ $\mathrm{O} \mathrm{cm}{ }^{2}, \bigcirc: C_{d l}^{* *}=1.0 \times 10^{-4} \mathrm{~F} / \mathrm{cm}^{2}, \times: C_{d l}^{* *}=2.0 \times 10^{-4} \mathrm{~F} / \mathrm{cm}^{2}$, $\square: C_{d l}^{* *}=3.0 \times 10^{-4} \mathrm{~F} / \mathrm{cm}^{2}$. The frequency range is 100 $\mathrm{mHz}-10 \mathrm{kHz}$.

two ranges is $\frac{R_{s o l}^{*} X}{3}$, and its value increases with the increase of $R_{s o l}^{*}$ in Fig. 8 (b). The impedance at transition frequency is identical in Figs. 8 (c) and (d) because the value of $R_{s o l}^{*}$ is same. The diameter of capacitive loop correspond to $\frac{R_{c t}^{* *}}{X L}$ and increases with the increase of $R_{c t}^{* *}$ in Fig. 8 (c). This result indicates that the locus is related to Faradaic process in the low frequency range since $\frac{R_{c t}^{* *}}{X L}$ is the charge transfer resistance at whole surface area of cylindrical electrode. The low frequency limiting value of impedance is total of solution resistance inside pore $\frac{R_{s o l}^{*} X}{3}$ and the charge transfer resistance $\frac{R_{c t}^{* *}}{X L}$ as presented in Eq. (36). In Fig. 8 (d), the shape of impedance is identical because the values of $R_{s o l}^{*}$ and $R_{c t}^{* *}$ are same. The frequency at maximum imaginary part corresponds to the time constant $R_{c t}^{* *} C_{d l \cdot}^{* *}$

3. 5. 1. 2 Complex capacitance The low frequency limiting value of complex capacitance is as follows.

$$
\begin{aligned}
& C_{\omega \rightarrow 0}^{\prime}=\frac{9 X R_{c t}^{* 2} C_{d l}^{*}}{\left(3 R_{c t}^{*}+R_{s o l}^{*} X^{2}\right)^{2}} \\
& =\frac{X L C_{d l}^{* *}}{\left(\frac{R_{c t}^{* *}}{X L}+\frac{R_{s o l}^{*} X}{3}\right)^{2} /\left(\frac{R_{c t}^{* *}}{X L}\right)^{2}}
\end{aligned}
$$

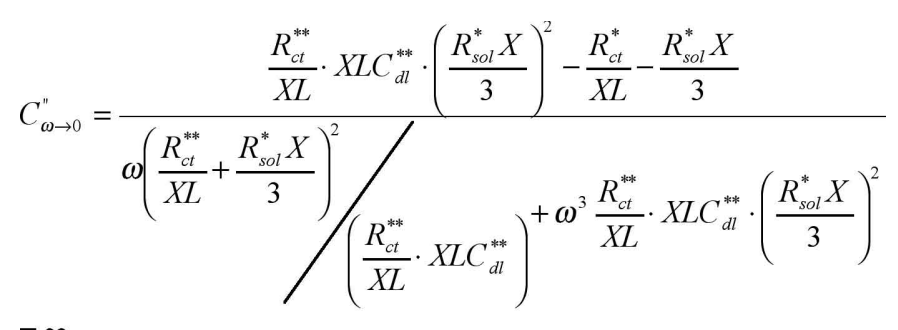

$=\infty$

The calculated results of complex capacitance with variations of $R_{s o l}^{*}, R_{c t}^{* *}$ and $C_{d l}^{* *}$ are shown in Figs. 8 (e), (f) and $(g)$, respectively. Complex capacitance describes the straight line of 45 degree to real axis in high frequency range, locus of semi-circle in middle frequency range and vertical line in low frequency range. In low frequency range, $C_{\omega \rightarrow 0}^{\prime}$ takes a constant value presented by Eq. (37). And the equation of $C_{\omega \rightarrow 0}^{\prime}$ is similar to that in Eq. (24), though the solution resistance $R_{\text {sol }}$ in Eq. (24) is replaced by $\frac{R_{s o l}^{*} X}{3}$. In Figs. 8 (e) - (g), $C_{\omega \rightarrow 0}^{\prime}$ changes by the variations of $R_{\text {sol }}^{*}, R_{c t}^{* *}$ and $C_{d l .}^{* *}$ Especially in Fig. $8(\mathrm{~g})$, the value of $C_{\omega \rightarrow 0}^{\prime}$ is in proportionate to the $C_{d l}^{* *}$ where $R_{s o l}^{*}$ and $R_{c t}^{* *}$ are constant values.

\section{6 The example of complex capacitance analysis of EDLC}

The typical experimental result of impedance of EDLC at the bias voltage of $2.0 \mathrm{~V}$ is shown in Fig. 9 (a). ${ }^{11)}$ Impedance spectrum can be divided into two frequency ranges, namely, the distributed constant range above 100 $\mathrm{mHz}$ and the lumped constant range below $100 \mathrm{mHz}$. The real part of impedance is constant, and the imaginary part increases with the decrease of the frequency at lumped constant range, indicating the blocking electrode behavior in the low frequency range. Impedance deviates from the locus of blocking electrode at distributed constant range. It is considered that the current distribution is non-uniform by porous structure of electrode in the high frequency range. The solution resistance at high frequency limit is approximately $0.002 \mathrm{O}$, indicating the low contact resistance and electrolyte resistance. The value of $Z^{\prime}$ at the limped constant range is $3.2 \times 10^{-3} \Omega$ where the value was subtracted by 0.002 O of $R_{\text {sol. }}$. From $Z^{\prime}=\frac{R_{s o l}^{*} X}{3}$, the solution resistance of pore 
(a)

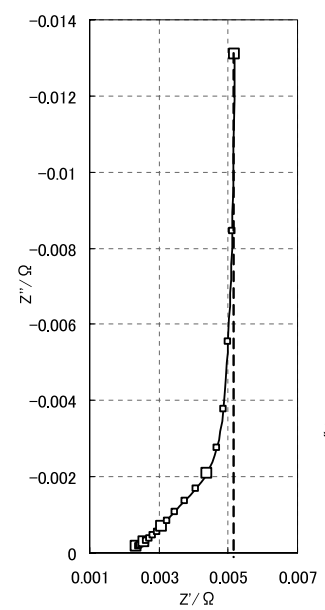

(b)
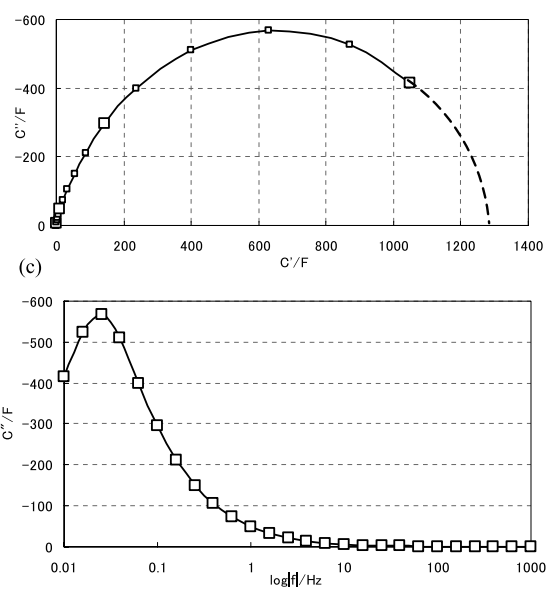

Fig. 9 Experimental result of electrochemical impedance of EDLC. The frequency range was $10 \mathrm{mHz}-1 \mathrm{kHz}$. The voltage was $2.0 \mathrm{~V}$ The temperature was $278 \mathrm{~K}$. (a) Electrochemical impedance, ${ }^{11)}$ (b) complex capacitance, and (c) imaginary capacitance plot.

in activated carbon electrode $R_{\text {sol }}^{*} X$ can be estimated $9.6 \times 10^{-3} \Omega$. In order to obtain the solution resistance of single pore, this value $9.6 \times 10^{-3} \Omega$ must be divided by the number of pores.

The calculated result of complex capacitance from Eq. (13) is shown in Fig. 9 (b). The $C$ describes semi-circle where the high frequency values are distorted. The extrapolating value of semi-circle $X L C_{d l}^{* *}$ in low frequency range means total capacitance of inner wall. In Fig. 9 (b), $X L C_{d l}^{* *}$ is estimated approximately $1300 \mathrm{~F}$. The $X L$ means effective surface area of total inner walls where great number of pores exists on the electrode surface. The value of $C_{d l}^{* *}$ was assumed as $3.0 \times 10^{-5} \mathrm{~F} / \mathrm{cm}^{2}$ from the reported values $3.0 \times 10^{-5} \mathrm{~F} / \mathrm{cm}^{2}$ by Candy et al. ${ }^{17)}, 3.0 \times$ $10^{-5} \mathrm{~F} / \mathrm{cm}^{2}$ by Srinivasan et al. ${ }^{18)}$ and $2.5 \times 10^{-5} \mathrm{~F} / \mathrm{cm}^{2}$ by Song et al. ${ }^{19)}$ The $X L$ was calculated as $4.3 \times 10^{7} \mathrm{~cm}^{2}$ at the bias voltage of $2.0 \mathrm{~V}$. The plots of imaginary capacitance and $\log |f|$ is shown in Fig. 9 (c) in order to compare the complex capacitance plot. Many researchers ${ }^{13-15)}$ is applied to analyze performance of EDLC because the time constant of charge/discharge can be estimated from the frequency at maximum peak of $C^{\prime \prime}$. Contrary to this, the complex capacitance plot gives the direct value $C_{\omega \rightarrow 0}^{\prime}$ from extrapolating value of semi-circle in the low frequency range in Fig. 9(b). In addition, the effective surface area can be estimated by be assumed $C_{d l}$.

\section{Conclusion}

Electrochemical impedance and complex capacitance spectra of typical equivalent circuits are calculated for analysis on ELDC. In the cases of equivalent circuits in Figs. 2 (a), 3 (a), 4 (a) and 7 (a), the $C_{d l}$ can be obtained from the real part of the complex capacitance directly in the low frequency range though the curve-fitting is necessary to calculate $C_{d l}$ from the impedance. The loci of the impedance and the complex capacitance with TLM were discussed in detail. Their loci were divided into the limped constant and distributed constant ranges in low and high frequency ranges, respectively. It was found that the complex capacitance allows the direct information concerning the capacitance of pore electorde.

\section{References}

1) K. S. Cole and R. H. Cole, J. Chem. Phys., 9, 341 (1941).

2) D. W. Davidson and R. H. Cole, J. Chem. Phys., 18, 1417 (1950) ; 19, 1418 (1951) ; 20, 1389 (1952).

3) C. Arbizzani, M. Mastragostino, and L. Meneghello, Electrochem. Acta. 144, 3066 (1997).

4) D. Qu and H. Shi, J. Power Sources, 74, 99 (1998).

5) P. L. Taberna, P. Simon, and J. F. Fauvarque, J. Electrochem. Soc., 150, A292 (2003).

6) C. Portet, P. L. Taberna, P. Simon, E. Flahaut, and C. Laberty-Robert, J. Electrochem. Soc., 50, 4174 (2005).

7) M. Eikering, A. A. Kornyshev, and E. Lust, J. Electrochem. Soc., 152, E24 (2005).

8) M. Itagaki, N. Kobari, S. Yotsuda, K. Watanabe, S. Kinoshita, and M. Ue, J. Power Sources, 135, 225 (2004).

9) M. Itagaki, N. Kobari, S. Yotsuda, K. Watanabe, S. Kinishida, and M. Ue, J. Power Sources, 148, 78 (2005).

10) M. Itagaki, S. Yotsuda, N. Kobari, K. Watanabe, S. Kinishida, and M. Ue, Electrochem. Acta., 51, 1629 (2006).

11) M. Itagaki, S. Suzuki, I. Shitanda, K. Watanabe, and H. Nakazawa, J. Power Sources, 164, 415 (2007).

12) J. H. Jang and S. M. Oh, J. Electrochem. Soc., 151, A571 (2004).

13) S. Yooh, J. H. Jang, B. H. Ka, and S. M. Oh, Electrochim. Acta, 50, 2255 (2005).

14) J. H. Jang, S. Yooh, B. H. Ka, Y. H. Jumg, and S. M. Oh, J. Electrochem. Soc., 152, A1418 (2005).

15) R. D. Levie, Electrochim. Acta, 8, 751 (1963).

16) J. P. Candy and P. Fouilloux, Electrochim. Acta, 26, 1029 (1981).

17) V. Srinivasan and J. W. Weidner, J. Electrochem. Soc., 146, 1650 (1999).

18) H. K. Song, H. Y. Hwang, K. H. Lee, and L. H. Dao, Electrochim. Acta, 45, 2241 (2000). 